\title{
Verification of a Polynomial Stochastic Fatigue Crack Growth Model
}

\author{
C. C. $\mathrm{Ni}$ \\ Department of Mechanical Engineering, China University of Science and Technology \\ Taipei 11522, Taiwan \\ cctommykimo@yahoo.com.tw
}

Keywords: Fatigue crack growth model; lognormal random process; 2024-T351

\begin{abstract}
The study is focused on the verifications of a proposed polynomial stochastic fatigue crack growth model, which assuming the fatigue crack growth rate equal to a deterministic polynomial function in terms of fatigue crack size multiplied by a stationary lognormal random factor accounting for the statistical scatter of the fatigue crack growth. Compact tension (CT) specimens cut from a 2024-T351 aluminum-alloy plate were used for fatigue crack growth tests under random loads performed on twenty-five specimens. The comparison of median crack growth curves was made between analytical and experimental outcomes. For extreme case of lognormal random variable, the comparisons of the fatigue crack growth curve, percentile fatigue crack growth curve, probability of crack exceedance, and distribution function of random time between analytical and experimental results were also investigated.
\end{abstract}

\section{Introduction}

Fatigue of engineering materials has been studied for over 160 years. It is a very complicated process that causes damage or failure of components or structures such as aircraft, bridges, and pressure vessels subject to dynamic loading, and is difficult to describe and model accurately. In spite of its complexities, fatigue damage assessment in the design of some mechanical components and structures still has to be made. As a result, fatigue analysis methods have been developed.

Based on engineering and macroscopic viewpoints, the mechanical properties of material are often considered homogeneous. However, an appreciable amount of scatter has been observed in fatigue experimental data even under the same testing condition. The result is attributed to the inhomogeneous material microstructure itself, e.g., random distribution of lattice defects, impurity atoms, slip systems, and crystal size and macro defects such as porosity, crack and casting defects. Therefore, the deterministic crack growth equations can't adequately describe fatigue crack growth behavior. As a result, probabilistic approaches for the fatigue crack growth have received great attention.

So far, many fatigue crack growth models have been proposed by researchers. Unfortunately, there is no unique and general fatigue crack growth equation to be used solely for describing the variety of fatigue crack growth phenomena. Some commonly used fatigue crack growth equations based on the fracture mechanics are the Paris-Erdogan law [1], Elber law [2], Forman law [3], and Walker law [4]. Approaches different from that based on fracture mechanics have also been proposed $[5,6]$. Of them, the most frequently used one is the power law in terms of crack size proposed by Yang et al. [5].

In this study, the investigations of a polynomial stochastic fatigue crack growth model based on the assumption that the fatigue crack growth rate equal to a deterministic polynomial function in terms of fatigue crack size multiplied by a stationary lognormal random factor accounting for the statistical scatter of the fatigue crack growth is proposed. The analytical results specially emphasized on the extreme cases of lognormal random variable were obtained. Comments on the results of verifications are made at the end of the paper. 


\section{Material, Specimen and Experiment}

Compact tension (CT) specimens cut from a 2024-T351 aluminum-alloy plate were used for fatigue crack growth tests. The chemical compositions of the material include Si $(0.07$ wt. \%), Fe $(0.11$ wt. \%), Cu (4.42 wt. \%), Mn (0.66 wt. \%), Cr (0.03 wt. \%), Ti (0.02 wt. \%), Zn (0.05 wt. \%) and Al (94.64 wt. \%). The mechanical properties of the material, nominal yield strength, ultimate strength and elongation, are $320 \mathrm{MPa}, 462 \mathrm{MPa}$ and $15.4 \%$ respectively. Fig. 1 shows the dimensions of the specimen.

Fatigue crack growth tests were performed on twenty-five specimens under random loads. The statistics of the random signals is tabulated in Table 1. Based on the ASTM standard E647-93 [7], the pre-cracking procedure was started at the beginning of test with a crack length of $15.00 \mathrm{~mm}$ counting from the loading line to the tip of v-shaped slot of specimen and extended to the length of $18.00 \mathrm{~mm}$. The crack length and its corresponding loading cycle were tracked after pre-cracking from $18.00 \mathrm{~mm}$ with 0 loading cycle to the instant specimen fractured for all twenty-five specimens. The experimental results, crack length versus loading cycle, of twenty-five specimens are shown in Fig.2.
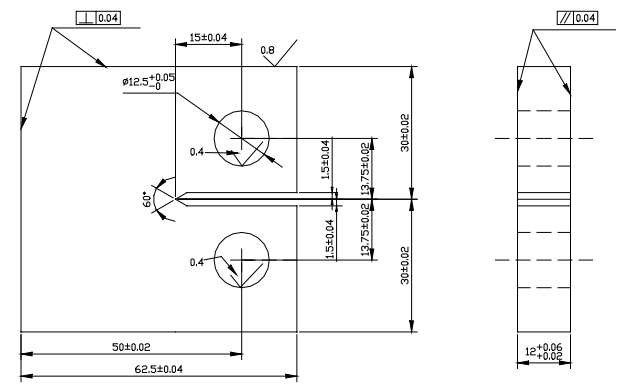

Fig. 1 Dimensions of CT specimen

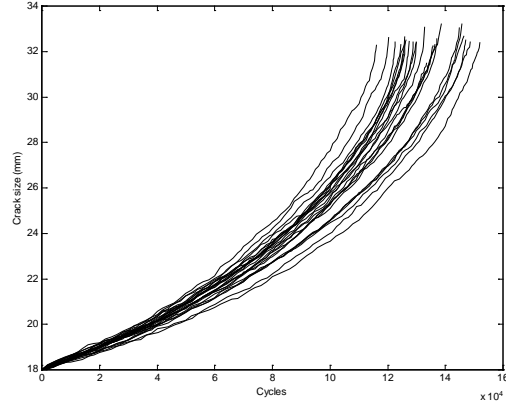

Fig. 2 Experimental fatigue crack growth curves

Table 1 Statistics of random signals

\begin{tabular}{lcccc}
\hline & All signal values & Peak values & Trough values & Amplitudes \\
\cline { 2 - 5 } Mean (N) & 5000 & 6118 & 3882 & 1118 \\
Std (N) & 1000 & 734 & 734 & 552 \\
\hline
\end{tabular}

\section{Polynomial Stochastic Fatigue Crack Growth Model}

A general lognormal random process equation, named polynomial stochastic fatigue crack growth model herein [6], is given as follows,

$$
\frac{d a(t)}{d t}=X(t)\left\{p+q a(t)+r[a(t)]^{2}\right\}
$$

where $a(t)$ is the crack length, $t$ can be either stress cycles, flight hours or flights depending on the applications, $p, q$ and $r$ are constants depending on the characteristics of the loading and materials, and $X(t)$ is a stationary lognormal random process with a median value of 1.0 and a standard deviation of $\sigma_{X}$. In other words, the random factor $Z(t)=\ln X(t)$ is a stationary normal random process with zero mean and standard deviation of $\sigma_{Z}$.

Rearranging and integrating Eq. 1 from initial crack size $a_{0}$ at time zero to crack size $a(t)$ at time $t$ results in 


$$
a(t)=\frac{(A-q) \sqrt{G}+(A q+G) \tan \frac{\sqrt{G} B(t)}{2}}{2 r\left[\sqrt{G}-A \tan \frac{\sqrt{G} B(t)}{2}\right]}
$$

where $G=4 p r-q^{2}, A=2 r a_{0}+q$, and $B(t)=\int_{0}^{t} X(\tau) d \tau$. Also, Rearranging and Integrating the deterministic crack growth equation, $\frac{d a(t)}{d t}=p+q a(t)+r[a(t)]^{2}$, the median service time to reach any specified crack size $a$ from initial crack size $a_{0}$ can be obtained as follows

$$
\bar{t}(a)=\int_{0}^{\bar{t}(a)} d t=\int_{a_{0}}^{a} \frac{1}{p+q \varsigma+r \varsigma^{2}} d \varsigma=\frac{2}{\sqrt{G}} \tan ^{-1} \frac{(2 r a-A+q) \sqrt{G}}{A q+G+2 r A a}
$$

As a function of $a, \bar{t}(a)$ is referred to as the median crack growth curve [6].

For the extreme condition referred to as lognormal random variable case, the lognormal random process $X(t)$ is completely correlated at any two instants of time. Under this condition, $X(t)$ becomes a lognormal random variable $X$ and hence the normal random process $Z(t)$ becomes a normal random variable $Z$. It results in $B(t)=\int_{0}^{t} X d \tau=X t$ and therefore Eq. 2 becomes as follows [6]

$$
a(t)=\frac{(A-q) \sqrt{G}+(A q+G) \tan \frac{\sqrt{G} X t}{2}}{2 r\left(\sqrt{G}-A \tan \frac{\sqrt{G} X t}{2}\right)}
$$

the crack growth curves can be simulated by substituting the random variables $X$ into Eq. 4, where $X=\exp (Z)$ and $Z$ are randomly generated normal random variables with a mean value of zero and standard deviation of $\sigma_{Z}$.

Also, the $\gamma$ percentile of the crack size, $a_{\gamma}(t)$, at service time $t$ follows from Eq. 4 can be expressed as follows

$$
a_{\gamma}(t)=\frac{(A-q) \sqrt{G}+(A q+G) \tan \frac{\sqrt{G} x_{\gamma} t}{2}}{2 r\left(\sqrt{G}-A \tan \frac{\sqrt{G} x_{\gamma} t}{2}\right)}
$$

where $x_{\gamma}$ is the $\gamma$ percentile of the random variable $X$ given by $x_{\gamma}=\exp \left(z_{\gamma}\right), z_{\gamma}$ is the $\gamma$ percentile of the normal random variable $Z$ given by $z_{\gamma}=\sigma_{Z} \Phi^{-1}\{1-\gamma \%\}$, and $\Phi^{-1}\{\}$ is the inverse standard normal distribution function [6]. The crack growth curve associated with value of $\gamma$ indicates the probability that the crack growth of a specimen in a data set will be faster than that of the curve is $\gamma \%$. 
In addition, the probability, which is referred to as probability of crack exceedance, that $a(t)$ will exceed any given crack size $a$ within the interval $(0, t)$ can be derived as [6]

$$
P_{a(t)}^{e}(a)=P[a(t) \geq a]=1-\Phi\left\{\frac{\ln \left[\frac{2}{t \sqrt{G}} \tan ^{-1} \frac{(2 r a-A+q) \sqrt{G}}{A q+G+2 r A a}\right]}{\sigma_{Z}}\right\}
$$

Similarly, the distribution function of random time $T(a)$ to reach a specified crack size $a$ from $a_{0}$ within the interval $(0, t)$, or the probability of $T(a)$ for crack size to reach any given value $a$, can also be derived as [6]

$$
F_{T(a)}(t)=P[T(a) \leq t]=1-\Phi\left\{\frac{\ln \left[\frac{2}{t \sqrt{G}} \tan ^{-1} \frac{(2 r a-A+q) \sqrt{G}}{A q+G+2 r A a}\right]}{\sigma_{Z}}\right\}
$$

\section{Numerical Verification}

Firstly, the median crack growth curve can be obtained by Eq. 3. The comparisons between analytical and experimental outcomes are shown in Fig. 3 and the results appear satisfactory. It is noted that the median crack growth curve obtained by Eq. 3 is the same as that of lognormal random variable case obtained from Eq. 5 for $\gamma=50$.

For the lognormal random variable case, the crack growth curves can be simulated by substituting the randomly generated variables $X$ into Eq. 4. Good correlations shown in Fig. 4 and Fig. 5 between the simulated curves and experimental data were found. Also, different $\gamma$ percentiles of the crack growth curves can be obtained directly through the use of Eq. 5. Again, good correlations between the analytical and experimental results of various percentiles are shown in Fig. 6. Finally, the probability of crack exceedance and the distribution function of random time and can be obtained through Eq. 7 and Eq. 8 respectively, and the results compared between analytical and experimental outcomes are displayed in Fig. 7 and Fig. 8. It is found that the analytical results can predict the experimental data quite well.

\section{Summary}

A polynomial stochastic fatigue crack growth model was investigated. Results presented in this study are specially emphasized on the verifications of the model for one extreme case, lognormal random variable case, through experimental fatigue crack growth data. Comments on the results of verifications are summarized as follows,

1. Using Eq. 3, the median crack growth curve can be verified.

2. For extreme case of lognormal random variable, Eq. 4, Eq. 5, Eq. 6, and Eq. 7 can be used to obtain the fatigue crack growth curve, percentile fatigue crack growth curve, probability of crack exceedance, and distribution function of random time, respectively.

3. The verified results between analytical outcomes obtained from the proposed polynomial stochastic fatigue crack growth model and experimental data appear satisfactory.

4. The comparisons amoung the proposed model and other models through experimental data will be performed in the further study. 


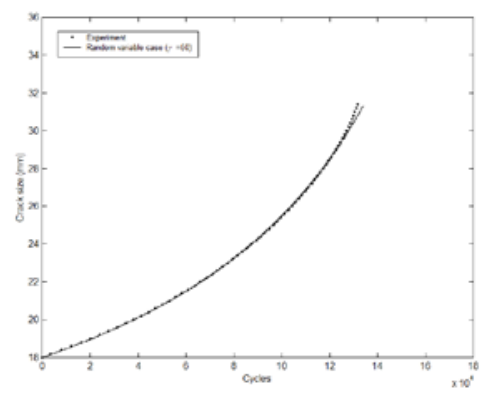

Fig. 3 Comparison of median crack growth curve

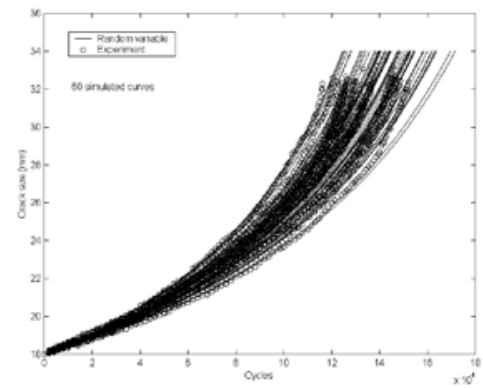

Fig. 5 Comparison of crack growth curves II
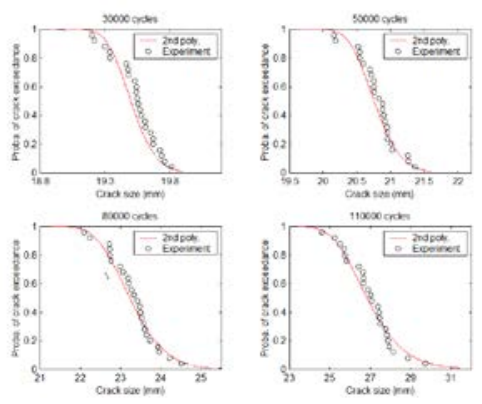

Fig. 7 Probability of crack exceedance

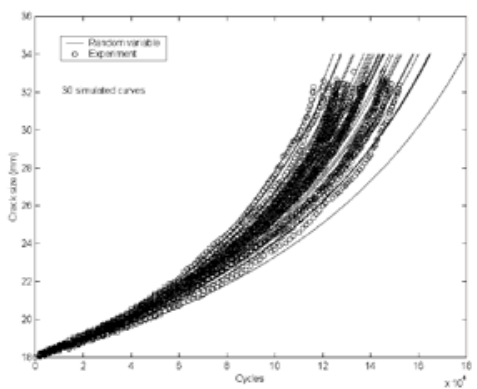

Fig. 4 Comparison of crack growth curves I

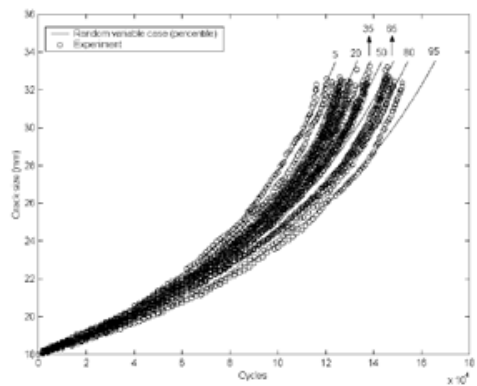

Fig. 6 Crack growth curves of various percentiles
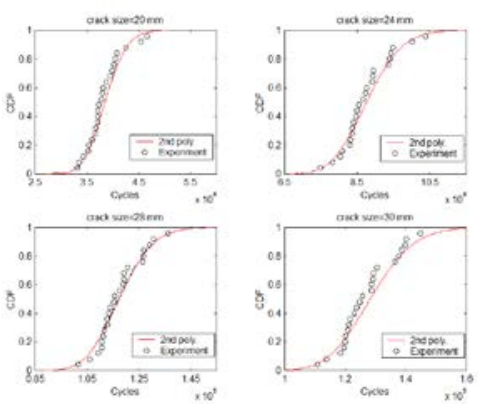

Fig. 8 Distribution of random time

\section{References}

[1] P.C. Paris and F. Erdogan: A Critical Analysis of Propagation Laws, Journal of Basic Engineering, Transactions of ASME, Vol. 85, (1963), pp. 528-534.

[2] W. Elber: The Stress Ratio Effect on Fatigue Crack Closure, Damage Tolerance of Aircraft Structures, ASTM STP 486, American Society for Testing and Materials, Philadelphia (1971).

[3] Forman, et al.: Numerical Analysis of Crack Propagation in Cyclic Load Structure, Journal of Basic Engineering, Transaction of ASME, Vol. 89 (1967), pp. 459-465.

[4] K. Walker: The Effect of Stress Ratio during Crack Propagation and Fatigue for 2024-T3 and 7075-T6 Aluminum, Effect of Environment and Complex Load History on Fatigue Life, ASTM STP 462, American Society for Testing and Material, Philadelphia (1970).

[5] J.N. Yang, S.D. Manning, and W.R. Garver: Durability Methods Development, Volume V-Durability Analysis Methodology Development, Technical Report AFFDL-TR-79-3118, Air Force Flight Dynamics Laboratory (1979).

[6] C.C. Ni: Formulation of a Polynomial Stochastic Fatigue Crack Growth Model, Advanced Materials Research, Vol. 909 (2014), pp. 467-471. 
[7] ASTM E647-91: Standard Test Method for Measurement of Fatigue Crack Growth Rates, American Society for Testing and Materials, Vol.03.01 (1991), pp. 679-706. 